\title{
Fuzzy State Space Model of Multivariable Control Systems
}

\author{
R. Ismail \\ Faculty of Information Technology and Quantitative Sciences \\ Universiti Teknologi MARA \\ 40450 Shah Alam, Selangor, Malaysia \\ Tel: 60-3-5544-2090Ｅ-mail: raisma2008@gmail.com \\ K. Jusoff (Corresponding author) \\ Yale University, Centre for Earth Observations \\ Environmental Science Centre, 21 Sachem St, New Haven, CT 06511, USA \\ Tel: 20-3-432-1384Ｅ-mail: jusoff.kamaruzaman@yale.edu \\ T. Ahmad \\ Faculty of Science, Universiti Teknologi Malaysia \\ 81310 Skudai, Johor, Malaysia \\ Tel: 60-7-553-4354 E-mail: tahir_22@hotmail.com \\ S. Ahmad \\ Faculty of Science, Universiti Teknologi Malaysia \\ 81310 Skudai, Johor, Malaysia \\ Tel: 60-12-771-3254 E-mail: sham@mathsun.utm.my \\ R.S. Ahmad \\ Faculty of Science, Universiti Teknologi Malaysia \\ 81310 Skudai, Johor, Malaysia \\ Tel: 60-12-737-2469 E-mail: shah@dfiz2.fs.utm.my
}

\begin{abstract}
Fuzzy State Space Model (FSSM) is a new modeling technique, which was developed for solving inverse problems in multivariable control systems. In this approach, the flexibility of fuzzy modeling is incorporated with the crisp state space models proposed in the modern control theory. The vagueness and uncertainty of the parameters are represented in the model construction, as a way of increasing the available information in order to achieve a more precise model of reality. Some important properties and characteristics of FSSM were also investigated. In this paper, our discussion is focused on the formulation of the FSSM that provides algorithms for optimization of input parameters directly. The effectiveness of this modeling approach is illustrated by implementing it to the state space model of a furnace system of a combined cycle power plant. The results obtained in this application demonstrate that the proposed new modeling approach is reasonable and provides an innovative tool for decision-makers.
\end{abstract}

Keywords: Fuzzy state space model, Inverse problems, Uncertainty, Fuzzy number

\section{Introduction}

The design of mathematical models of complex real-world systems is essential in many fields of science and engineering. A common approach is to assume that the structure of the model is given directly as a parameterized mathematical 
function, which is based on physical principles. However, for many real-world systems a great deal of information is provided by human experts, who describe the system verbally through vague, uncertain or imprecise statements. The fact that humans are often able to manage complex tasks under significant uncertainty has stimulated the search for alternative modeling and control paradigms. A typical example of techniques that make use of human knowledge and deductive processes is fuzzy modeling. Even then, most of the systems considered in literatures are single-input single-output (SISO) or multi-input single-output (MISO) systems.

Due to the complexity of most practical multivariable or multi-input multi-output (MIMO) control systems, it is necessary to develop a mathematical model of the systems by simplifying and idealizing the processes involved. Control system analysis normally addresses forward problems. However, disturbance in power systems motivate analysis questions that are classed as inverse problems (Hensel, 1991). Traditionally, such inverse problems have been addressed by repeated simulation of forward problems, for example Ordys et al (1994), Ram \& Patel (1998). Thus, the objective of this paper is to present the formulation of a new modeling technique, known as Fuzzy State Space Model (FSSM). FSSM provides an algorithm that address inverse problems in multivariable control systems directly. In this approach, the flexibility of fuzzy modeling is incorporated with the crisp state space models proposed in the modern control theory. The state space formulation has been a convenient basis for the development of advanced multivariable controller design methodologies (Ogata, 1997). This is because the underlying time-domain models, which are the most natural description of most problems of interest, can address a more general class of problem definition. Besides, the state variable model of a system includes a description of the internal status of the system, in addition to the input-output behavior. To take into account the uncertainties in the model, the uncertain value parameters of the system to be controlled are represented by fuzzy numbers (Kaufmann \& Gupta, 1985) with their membership function derived from expert knowledge.

The paper is organized as follows. After this introduction, section 2 describes the approaches in constructing the FSSM of multivariable control system which consider the mental, verbal and mathematical models. The development of the FSSM is described in section 3. The formulation of the Fuzzy State Space algorithm for determining the optimal parameter estimation is explained in Section 4. The validity of this algorithm is shown by implementing it to the state space model of a furnace system with three input parameters, which is presented in section 5. Finally, section 6 draws some conclusions from the presented work.

\section{Approaches in model construction}

A critical step in the application of model-based control algorithm is the development of a suitable model of the process dynamics. To effectively develop models, we need to blend information of different nature: experience of experts and operators, measurements and first principle knowledge formulated by mathematical equations. Thus, in the knowledge-based construction of the FSSM, the three different kinds of models considered are the mental model, verbal model and the mathematical model. From experience, intuition and expert knowledge, we build mental model in our mind. The verbal model is then formulated using "If...then..." rules, which is a very common means of description in everyday life. The verbal model can also be formulated based on fuzzy or uncertain descriptions such as "about 15", "almost 40", "around 600". The uncertain value parameters of the system are represented by triangular fuzzy numbers (TFN) that are used to analyze and manipulate approximate numeric values. TFN are used as they have an intuitive appeal and are easily specified by experts (Pedrycz, 1994). Thus, fuzzy sets serve as a smooth interface between qualitative variables and numerical domains of the inputs and outputs of the model.

For system analysis and engineering purposes, mathematical models are often constructed, for instance based on algebraic and differential or difference equations which are derived from physical laws. For well-defined systems, these standard mathematical tools lead to good models, even though the modeling process is often very tedious. However, most of the real-world systems are complex and nonlinear. Analytical approach for such systems is available only to a very limited extend (Bossel, 1994). On the other hand, a well-developed set of analytical tools is readily available for linear systems. Thus, linearization of nonlinear systems into linear state space model plays an important role. The most important advantage of the crisp state space model is that the system dynamic properties are condensed in the model (Cao \& Rees, 1995). The system model gives both the external and its internal behaviour of the system. Therefore, FSSM can be seen as a modeling framework for blending information of different nature, qualitative as well as quantitative. It can adequately process not only the given data, but also the associated uncertainty.

There are two important facts that make this modeling approach intuitively appealing. Firstly, there are always uncertain factors affecting the system in a real-world modeling situation. This indicates that a complete physical model can hardly be constructed. However, uncertain factors can be taken care by employing sufficiently flexible model. Secondly, the restriction on the flexibility to comply with the prior knowledge is allowed in the modeling procedure.

\section{Development of fuzzy state space model}

In developing Fuzzy State Space Model (FSSM), the advantages of the white-box and black-box modeling approach are 
combined. This means that the known parts of the system are modeled using physical knowledge, and the unknown or less certain parts are approximated using process data and black-box modeling structures with suitable approximation properties. Hence, FSSM exhibits some properties of the grey-box techniques. However, a great deal of information for many real-world systems, are provided by human experts who describes the system verbally through vague, uncertain or imprecise statements. Thus, the concepts from fuzzy sets (Zadeh, 1965) are used in the specification of the system's parameters, in which the parameters are fuzzy numbers instead of crisp numbers. It is interesting to note that, in this approach the input parameters can be described as approximately as desired at the early stages of the control process. This is an advantage as any early decision can restrict the set of available alternatives. The approximate description in term of uncertain input parameters is used to calculate the corresponding approximate characterization of relevant output parameters, which is then utilized to determine the optimal input parameters.

It is assumed that the multivariable dynamic system can be transformed into a solvable state space model. In state space model, the system dynamics properties are condensed in the model, which reflects its most important advantage. Besides that, it is also assumed that there is no direct transmission between the input parameters and the output parameters. Thus, FSSM of a multivariable dynamic system is defined as follows:

Definition: A Fuzzy State Space Model of a multivariable dynamic system is defined as

$S_{g F}: \quad \dot{\boldsymbol{x}}(t)=A \boldsymbol{x}(t)+B \tilde{\boldsymbol{u}}(t)$

$\tilde{\boldsymbol{y}}(t)=C \boldsymbol{x}(t)$

where $\tilde{u}$ denotes the fuzzified input vector $\left[u_{l}, u_{2}, \ldots, u_{n}\right]^{\mathrm{T}}$ and $\tilde{y}$ denotes the fuzzified output vector $\left[y_{1}, y_{2}, \ldots, y_{m}\right]^{\mathrm{T}}$ with initial conditions as $t_{0}=0$ and $\boldsymbol{x}_{0}=\boldsymbol{x}\left(t_{0}\right)=0$. The elements of state matrix $A_{p \times p}$, input matrix $B_{p \times n}$, and output matrix $C_{m \times p}$ are known to a specified accuracy.

The development of the algorithm for FSSM is based on three phases of a fuzzy system. Figure 1 shows these phases as fuzzification, fuzzy environment and defuzzification. In the first phase, each of the uncertain input parameters is fuzzified by specifying its $\alpha$-cuts. For each $\alpha$-cut, the possible combinations of the endpoints interval are used to calculate the induced performance parameters and the desired output parameters. All these parameters are processed by the Zadeh's extension principle (Klir \& Yuan, 1995) in the second phase, to determine the associated fuzzy value that is represented by the intersection between the induced performance parameters and the desired output parameters. In the final phase, the fuzzy value is defuzzified in order to obtain the valid combination of the input parameters. Subsequently, the optimal combination of the input parameters is determined by the Extension of Optimized Defuzzified Value Theorem.

\section{$<<$ Figure 1. Phases in developing fuzzy algorithm>>}

\section{Formulation of fuzzy state space algorithm}

According to Babuska and Verbruggen (1996), MIMO systems can be represented in a decomposed form as a set of coupled MISO models. Thus, the global modeling problem can be reduced to the development of fuzzy MISO models with $n$ inputs and one output. This idea is undertaken by the formulation of the FSSM for MISO systems (Ismail et al., 2004). However, the MIMO fuzzy model which is constructed based on a group of MISO fuzzy models, will result in an increased burden of computation time (Wang, 1994). Efforts in developing a model for solving a MIMO system directly will certainly be an advantage, especially in terms of time and cost. For this reason, the Fuzzy State Space algorithm for a MISO system is enhanced to accommodate a MIMO system.

Given an input $u_{i}$ that takes values in set $I_{i}$, and let preferences for different values of $u_{i}$ be expressed by a fuzzy set $F_{I i}$ on $I_{i}$. For each $x \in I_{i}$, the value $F_{I i}(x)$ designates the degree of desirability of using the particular value $x$ within the given set of values $I_{i}$. Thus, set $F_{I i}$ is referred to as the set of desirable values of parameter $I_{i}$, and $F_{I i}(x)$ is viewed as the grade of membership of value $x$ in this set. Index $i$ is used here to distinguish different input parameters.

The fuzzy sets expressing preference for all input parameters are employed for calculating the associated fuzzy sets for performance parameters. The target values of performance parameters are specified by functional requirements. Performance parameters, resulting from calculations with imprecise or vague input parameters, will also be represented by fuzzy preference functions. Similarly, each of the output parameters is represented by a range and a preference function. It is assumed that all the fuzzy sets $F_{I i}$ expressing preferences of all input parameters $u_{i} \in I_{i} \subset \Re(i \in \aleph)$ are determined, normalized and convex. $I$ is a close interval of real numbers. $S_{g F}$ is a performance parameter based on the FSSM whereby all input parameters are considered as its variables and can be presented within a fuzzy set $F_{S g F}$. The algorithm to determine $F_{\text {ind }}$, a fuzzy set that is induced on the performance parameters $S_{g F}$ has the following steps:

Step 1: Let $\quad S_{g F}: \Re^{n} \rightarrow \mathfrak{R}^{m} . S_{g F}$ is the performance parameter based on FSSM such that $\left(r_{1}, r_{2}, r_{3}, \ldots, r_{m}\right) \stackrel{g F}{=} \dot{S}_{g F}\left(u_{1}, u_{2}, u_{3}, \ldots, u_{n}\right)$

Step 2: Select appropriate values for $\alpha$-cut such that $\alpha_{1}, \alpha_{2}, \alpha_{3}, \ldots, \alpha_{k} \in[0,1]$ which are equally spaced. 
Step 3: To fuzzify the input, determine all the $\alpha_{k}$-cuts for all $F_{I i}(i \in \aleph)$.

Step 4: Generate all $2^{n}$ combinations of the endpoints of intervals representing $\alpha_{k}$-cuts for all $F_{I i}(i \in \mathfrak{\aleph})$. Each combination is an $n$-tuple $\left(u_{1}, u_{2}, u_{3}, \ldots, u_{n}\right)$.

Step 5: Determine $\left(r_{1}, r_{2}, r_{3}, \ldots, r_{m}\right)_{\alpha_{i}}=S_{g F}\left(u_{1}, u_{2}, u_{3}, \ldots, u_{n}\right)_{\alpha_{i}}$ for each $n$-tuple and for all $i=1,2, \ldots, k$.

Step 6: With respect to $\alpha_{i}$, find $\min \left(r_{j}\right)_{\alpha_{i}}=r_{j}\left(\alpha_{i(\min )}\right)$ and $\max \left(r_{j}\right)_{\alpha_{i}}=r_{j}\left(\alpha_{i(\max )}\right)$

Set $\left(F_{\text {ind }}\right)_{\alpha_{i}}=\left[\min \left(r_{j}\right)_{\alpha_{i}}, \max \left(r_{j}\right)_{\alpha_{i}}\right]$ where $i=1,2, \ldots, k$ and $j=1,2, \ldots, m$.

Step 7: Determine all the $\alpha_{k}$-cuts for all the desired performance parameter $F_{S g F}$.

Step 8: Generate all $2^{m}$ combinations of the endpoints of intervals representing $\alpha_{k}$-cuts for all $F_{S g F}$. Each combination is an $m$-tuple. In this case, $m=2$.

Step 9: Set $\left[F_{\text {ind }} \wedge F_{S g F}\right]$.

Step 10: Determine $f_{j}^{*}=\sup \left[F_{\text {ind }} \wedge F_{S g F}\right]$ for all $j$ and find $S_{g F}{ }^{*}$, the $S_{g F}$ value of $f_{j}^{*}$.

Step 11: Find the endpoints of interval for each input $F_{I i}$ where $i=1,2,3, \ldots, n$.

Step 12: Generate all $2^{n}$ combinations of the endpoints of intervals representing $f^{*}$ - cuts for all $F_{I i}\left(i \in \aleph^{\aleph}\right)$. Each combination is an $n$-tuple $\left(u_{1}{ }^{*}, u_{2}{ }^{*}, u_{3}{ }^{*}, \ldots, u_{n}{ }^{*}\right)$.

Step 13: Determine $r_{j}^{*}=S_{g F}{ }^{*}\left(u_{1}, u_{2}, u_{3^{\prime}}, \ldots, u_{n}\right)_{f j^{*}(o p t)}$ by using the Extension of Optimized Defuzzified Value Theorem; which is stated below

Let $S_{g F}: \mathfrak{R}^{n} \rightarrow \mathfrak{R}^{m}$ where $S_{g F} \quad$ is a performance parameter based on the FSSM.

(a) If $S_{g F^{*}}{ }^{*}=r_{j}{ }^{*}=\max r_{j}$ such that $\mu\left(r_{j}{ }^{*}\right)=f^{*}$ for all $\left(r_{j}, f^{*}\right) \in F_{\text {ind }}$, then $r_{j}{ }^{*}=S_{g F}{ }^{*}=\max \left\|S_{g F}\left(u_{1}{ }^{*}, u_{2}{ }^{*}, \ldots, u_{n}{ }^{*}\right)\right\|$ where $\mu\left(u_{i}^{*}\right)=f^{*}$ for $i=1,2,3, \ldots, n$.

(b) If $S_{g F^{*}}{ }^{*}=r_{j}^{*}=\min r_{j}$ such that $\mu\left(r_{j}^{*}\right)=f^{*}$ for all $\left(r_{j,} f^{*}\right) \in F_{\text {ind }}$, then $r_{j}^{*}=S_{g F}{ }^{*}=\min \left\|S_{g F}\left(u_{1}^{*}, u_{2}{ }^{*}, \ldots, u_{n}{ }^{*}\right)\right\|$ where $\mu\left(u_{i}^{*}\right)=f^{*}$ for $i=1,2,3, \ldots, n$.

The theorem indicates that if the fuzzy preferred or desired parameter intersects on the maximum side of the fuzzy induced parameter, then the set of optimized parameters is the set for the maximum norm of the induced values. On the other hand, if the fuzzy desired parameter intersects on the minimum side of the fuzzy induced parameter, then the set of optimized parameters is the set for the minimum norm of the induced values. Thus, this theorem enables the decision-maker to identify the best-optimized value from predicated results in the final phase of the algorithm. It has been shown that all normal and convex fuzzy sets $F_{l i}$, expressing preferences of all input parameters $g_{i} \in I_{i} \subset R^{+}(i \in N)$ are mapped by the FSSM into the normal and convex induced fuzzy sets (Ismail et al., 2002).

\section{Implementation to a furnace system}

To illustrate the implementation of the Fuzzy State Space algorithm for MIMO system, we refer to the state space model of the furnace system developed in Ismail et al., (2005). We consider the two output parameters of the furnace system, $Q_{e s}$ (heat transferred to the economizers in $\mathrm{J} / \mathrm{s}$ ) and $p_{G}$ (furnace air pressure in $\mathrm{Pa}$ ). $Q_{e s}$ and $p_{G}$ are also the output parameters for the boiler system of the combined cycle power plant (Ordys et al., 1994). The implementation of the Fuzzy State Space algorithm with MIMO structure is discussed according to the three phases of fuzzy system, that is, fuzzification of parameters, processing of fuzzified parameters in the fuzzy environment and defuzzification of results.

Phase 1: Fuzzification

Each of the input parameter of the furnace system is fuzzified. The desired value for each input parameter has a value $\alpha=$ 1 whereas the domain or the extreme values are specified as $\alpha=0$ as shown in Table 1 .

\section{$<<$ Table 1: Input parameters specification>>}

In this illustration, $\alpha$-cuts with increment of 0.2 are used to calculate $F_{\text {ind }}$, the fuzzy values of induced output or performance parameters. Based on the steady state operating data (Ordys et al., 1994), each output parameters can be expressed as a linear combination of the input parameters. Using similar domain and desired values of the input parameters, each of the input parameter is fuzzified. $\alpha$-cuts with increment of 0.2 are used to calculate $F_{\text {ind }}$, the fuzzy values of induced output or performance parameters. Combinations of the endpoints of intervals for all input parameters with respect to each particular value of $\alpha$-cut are determined. The number of combinations increases with a smaller value of the $\alpha$-cut. The induced performance parameter $F_{S g}$ is determined by taking the maximum and minimum value of each performance parameter. These values are used to plot the graph of $F_{S g}$.

Similarly each of the desired output parameter is set to the values published in Ordys et al. (1994), which are obtained through forward calculations. The values for the output parameters are

$$
Q_{e s}=\left[1.2 \times 10^{6} ; 1.2465 \times 10^{6} ; 1.4 \times 10^{6}\right] \text { and } P_{G}=\left[9.0 \times 10^{4} ; 1.013 \times 10^{5} ; 1.2 \times 10^{5}\right]
$$


These values are used to calculate the preferred performance parameters. $\alpha$-cuts with an increment of 0.2 as in the fuzzification of input parameters are used to calculate $F_{S g F}$, the fuzzy values of preferred or desired output parameters.

Phase 2: Fuzzy environment

The intersection of the fuzzy preferred output parameter and the fuzzified performance parameter is determined by superimposing the two graphs in order to obtain the $f^{*}$ - value as shown in Figure 2. The largest fuzzy membership value, $f_{j}^{*}$, is taken if there are more than one intersection points.

Phase 3: Defuzzification

For output parameters $Q_{e s}$ and $p_{G}$ of the furnace system, the fuzzy value is computed to be $f^{*}=0.8141$. With the $f^{*}$ value obtained, the steps in the defuzzification process are carried out to calculate the best possible combination of the input parameters in order to accommodate the constraints defined in the process of fuzzification. With three imprecise or uncertain input parameters, there are eight possible combinations of the endpoints of interval. Each of these combinations is then substituted in the performance parameter.. The optimized input parameters are determined by using the Extension of Optimized Defuzzified Value Theorem

\section{$<<$ Figure 2. Fuzzy value for Furnace system $\left(Q_{e s}\right.$ and $\left.p_{G}\right)>>$}

The results of implementation the Fuzzy State Space algorithm for a MIMO furnace system are shown in Table 2, where the optimal input parameters estimation are $w_{F}=12.7436 \mathrm{~kg} / \mathrm{s}, w_{A}=65.9296 \mathrm{~kg} / \mathrm{s}$ and $w_{G}=22.5577 \mathrm{~kg} / \mathrm{s}$. These values differ from the desired values with an error of about $6.19 \%, 1.43 \%$ and $2.53 \%$ respectively. Using this calculated input values, the percentage error for the output parameters of the furnace system, $Q_{e s}$ and $p_{G}$, is computed to be $2.21 \%$ and $2.07 \%$ respectively. It is interesting to note that the calculated values obtained using this algorithm are very close to the desired target values of the system.

\section{$<<$ Table 2. Optimized input parameters $>>$}

Subsequently, a comparison is made between the optimal input parameters obtained using the Fuzzy State Space algorithm and the result obtained through simulation carried out in Ordys et al. (1994). The percentage error is calculated and tabulated in Table 3. The aim of this comparison is to highlight the difference between inverse modeling by utilizing fuzzy sets and a widely accepted forward modeling based on simulation. With the TFN used in modeling the uncertainty, the obtained result should have the same value as the result in Ordys et al. (1994) with no uncertainty consideration. It is observed that the values of the input parameters $w_{F}$ (fuel flow to the furnace in $\mathrm{kg} / \mathrm{s}$ ), $w_{A}$ (air flow to the furnace in $\mathrm{kg} / \mathrm{s}$ ), and $w_{G}$ (exhaust gas flow from the gas turbine in $\mathrm{kg} / \mathrm{s}$ ) differ with an error of $9.51 \%, 2.86 \%$ and $2.63 \%$ respectively. The determination of the optimal input parameters subjected to the desired output parameters can be obtained in a few computer runs, as compared to several hundreds computer runs that is required for the commonly accepted forward simulation approach. In order to properly model the uncertainties and further improve the results, the parameters of the fuzzy numbers which are used to model uncertainties in this study, need to be adjusted based on the historical data or human experience. For a better resolution, $\alpha$-cuts with much smaller increment can be used.

The good results obtained in this application show that this approach may become an interesting tool for decision-makers. Besides, it is relatively easy to take into account experts knowledge and considerations for establishing the membership functions.

\section{Conclusion}

The formulation of the FSSM for multivariable control system was presented. The construction of this model involved the integration of three different kinds of models, namely mental model, verbal model, and mathematical model. TFN are used to represent imprecise or uncertain parameters in the model, with their membership function derived from expert knowledge. The procedure in the Fuzzy State Space algorithms involved fuzzification of all the input parameters to create fuzzy environment. This is then processed to produce the induced output parameters. The best input parameters were extracted through defuzzification using an important theorem, Extension of Optimized Defuzzified Value Theorem.

Although we have illustrated the implementation of FSSM for the furnace system of combined cycle power plant, it can be applied to any multivariable control system as long as the mathematical model of the system can be expressed in state space representation. The influence of the initially assumed uncertainties on the overall solution of the problem is reflected in the results. The determination of the optimal input parameters estimation subjected to the desired output parameters can be obtained in a few computer runs, as compared to several hundred computer runs that are required for the commonly accepted forward simulation approach. Besides that, the performance of these algorithms can be further improved by changing the initial input parameters or by reducing the $\alpha$-cut increment. In general, this new technique for determination of optimal input parameters gives a broader and useful information and provides a faster and innovative tool for decision-makers. 


\section{References}

Babuska, R. \& Verbruggen, H.B. (1996). An overview of Fuzzy Modeling for Control. Control Eng. Practice. 4(11): $1593-1606$.

Bossel, H. (1994). Modeling and Simulation. Wellesley, M.A.: AK Peters Ltd.

Cao, S.G. \& Rees, N.W. (1995). Identification of Dynamic Fuzzy System. Fuzzy Sets and Systems. $74: 307$ - 320.

Hensel, E. (1991) Inverse Theory and Applications for Engineers. Eaglewood Cliffs, N.J.: Prentice Hall.

Ismail, R., Ahmad, T., Ahmad, S., \& Ahmad, R.S. (2002). Cembung dan Normal Teraruh menerusi Pemodelan Keadaan-Ruang Kabur, Prosiding SKSM ke-10, UTM Malaysia. 23-24 Dec 2002. 34 - 37.

Ismail, R., Ahmad, T., Ahmad, S., \& Ahmad, R.S. (2004). An Inverse Fuzzy State Space Algorithm for Optimization of Parameters in a Furnace System, Proceeding of the Joint $2^{\text {nd }}$ Int. Conf. on Soft Computing and Intelligent Systems and $5^{\text {th }}$ Int. Conf. Symposium on Advanced Intelligent Systems (SCIS-ISIS2004), Yokohama, Japan. 21 - 24 Sept. 2004.

Ismail, R., Ahmad, T., Ahmad, S., \& Ahmad, R.S. (2005). State Space Modeling of a Furnace systems. Prosiding SeminarMatematik 2005, FTMSK, Universiti Teknologi MARA, Malaysia.

Kaufmann, A. \& Gupta, M.M (1985). Introduction to Fuzzy Arithmetic: Theory and Applications, New York: Van Nostrand Reinhold Co.

Klir, G. J. \& Yuan, B. (1995) Fuzzy Sets and Logic: Theory and Applications. New Jersey: PTR Prentice Hall.

Ogata, K. (1997). Modern Control Engineering. $3^{\text {rd }}$ ed. Upper Saddle River: Prentice-Hall International.

Ordys, A.W., Pike, A.W., Johnson, M.A., Katebi, R.M. \& Grimble, M.J. (1994). Modelling and Simulation of Power Generation Plants. London: Springer-Verlag..

Pedrycz, W. (1994). Why Triangular Membership Functions?. Fuzzy Sets and Systems. 64: 21 - 30.

Ram, B. \& Patel. G.. (1998). Modelling Furnace Operation using Simulation and Heuristics. Proceedings of the 1998 Winter Simulation Conference. 957 - 963.

Wang, L.X. (1994). Adaptive Fuzzy Systems and Control: Design and Stability Analysis. Englewood Cliffs, N.J: PTR Prentice-Hall.

Zadeh, L.A. (1965). Fuzzy Sets, Information and Control, 8(3), 338 - 353, 1965.

Table 1. Input parameters specification

\begin{tabular}{llll}
\hline input parameters & $\alpha=0$ & $\alpha=1$ & $\alpha=0$ \\
\hline$w_{F}$ & 10 & 12 & 16 \\
$w_{A}$ & 60 & 65 & 70 \\
$w_{G}$ & 20 & 22 & 25 \\
\hline
\end{tabular}

Table 2. Optimized input parameters

\begin{tabular}{llll}
\hline$f^{*}=0.8141$ & Calculated Values & Desired Values & Error $(\%)$ \\
\hline$w_{F}$ & 12.7436 & 12 & 6.19 \\
$w_{A}$ & 65.9296 & 65 & 1.43 \\
$w_{G}$ & 22.5577 & 22 & 2.53 \\
\hline
\end{tabular}

Table 3. Comparison of optimized input parameters

\begin{tabular}{cccc}
\hline Input Parameters & Ismail's & Ordys et al. & difference (\%) \\
\hline$w_{F}$ & 12.7436 & 14.083 & 9.51 \\
$w_{A}$ & 65.9296 & 64.093 & 2.86 \\
$w_{G}$ & 22.5577 & 23.168 & 2.63 \\
\hline
\end{tabular}




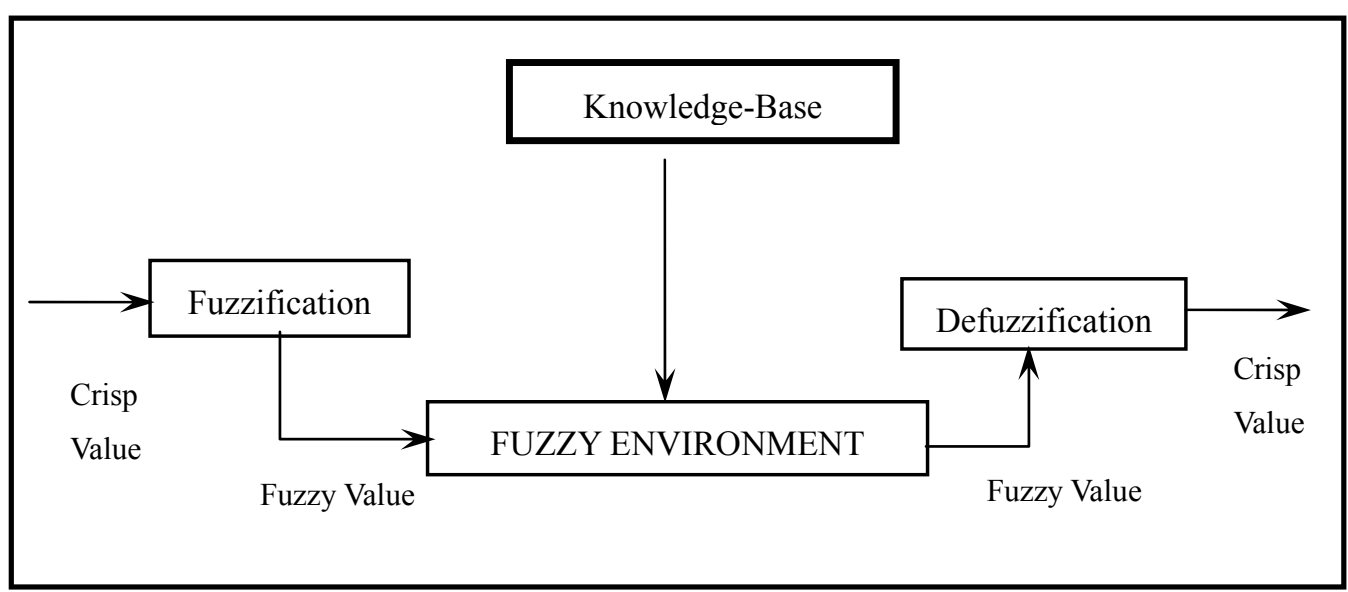

Figure 1. Phases in developing fuzzy algorithm

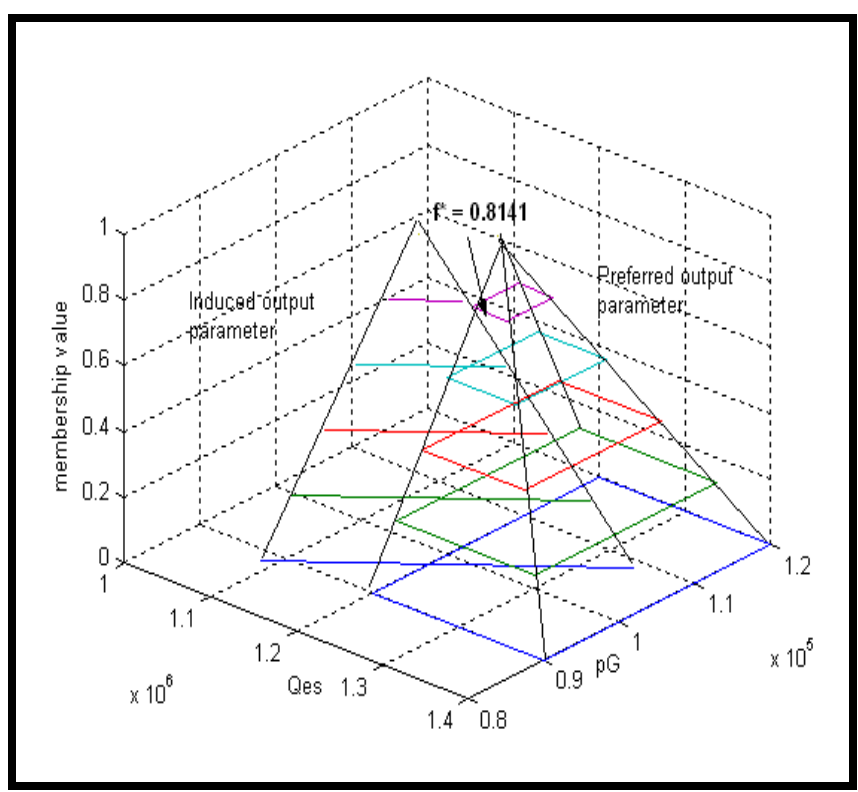

Figure 2. Fuzzy value for Furnace system $\left(Q_{e s}\right.$ and $\left.p_{G}\right)$ 